\title{
Identification of key microRNAs and target genes for the diagnosis of bone nonunion
}

\author{
YUAN XIONG $^{1 *}$, FAQI CAO ${ }^{1 *}$, LANG CHEN $^{1}$, CHENCHEN YAN $^{1}$, WU ZHOU $^{1}$,

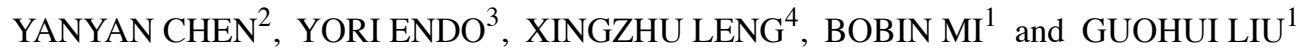

\author{
${ }^{1}$ Department of Orthopedics, Union Hospital, Tongji Medical College, Huazhong University of Science and Technology, \\ Wuhan, Hubei 430022; ${ }^{2}$ Department of Pathology, Zhongnan Hospital of Wuhan University, Wuhan, Hubei 430071, \\ P.R. China; ${ }^{3}$ Division of Plastic Surgery, Brigham and Women's Hospital, Harvard Medical School, Boston, MA 02215, USA; \\ ${ }^{4}$ Department of Biomedical Sciences, UMC Utrecht, Utrecht University, Utrecht, 3508 GA, The Netherlands
}

Received August 6, 2019; Accepted December 12, 2019

DOI: $10.3892 / \mathrm{mmr} .2020 .10996$

\begin{abstract}
A number of recent studies have highlighted the causes of bone nonunion (BN), however, the rate of BN incidence continues to rise and available therapeutic options to treat this condition remain limited. Thus, to prevent disease progression and improve patient prognosis, it is vital that $\mathrm{BN}$, or the risk thereof, be accurately identified in a timely manner. In the present study, bioinformatics analyses were used to screen for the differentially expressed genes (DEGs) and differentially expressed miRNAs (DEMs) between patients with $\mathrm{BN}$ and those with bone union, using data from the Gene Expression Omnibus database. Furthermore, clinical samples were collected and analyzed by reverse transcription-quantitative PCR and western blotting. In vitro and in vivo experiments were carried out to confirm the relationship between $\mathrm{BN}$ and the DEGs of interest, in addition to being used to explore the underlying molecular mechanism of BN. Functional enrichment analysis of the downregulated DEGs revealed them to be enriched for genes associated with 'ECM-receptor interactions', 'focal adhesion', 'and the calcium signaling pathway'. When comparing DEM target genes with these DEGs, nine DEGs were identified as putative DEM targets, where hsa-microRNA (miR)-1225-5p-CCNL2, hsa-miR-339-5p-PRCP, and hsa-miR-193a-3p-mitogen-activated protein kinase 10 (MAPK10) were the only three pairs which were associated with decreased gene expression levels.
\end{abstract}

Correspondence to: Professor Guohui Liu or Dr Bobin Mi, Department of Orthopedics, Union Hospital, Tongji Medical College, Huazhong University of Science and Technology, 1277 Jiefang Road, Wuhan, Hubei 430022, P.R. China

E-mail: liuguohui@medmail.com.cn

E-mail: mi19882@163.com

${ }^{*}$ Contributed equally

Key words: biological markers, bioinformatics, gene array, protein array, microRNA
Furthermore, hsa-miR-193a-3p was demonstrated to induce BN by targeting MAPK10. Collectively, the results of the present study suggest that hsa-miR-193a-3p may be a viable biomarker of $\mathrm{BN}$.

\section{Introduction}

Bone nonunion (BN) is a frequent complication following bone fracture, occurring when fracture healing ceases without suitable bone union (1-3). While much research is required to fully elucidate the causes of $\mathrm{BN}$ (4), the rate of $\mathrm{BN}$ incidence continues to rise, with $\leq 10 \%$ of bone fracture patients estimated to have suffered from BN (5). It is thus vital that $\mathrm{BN}$, or the risk thereof, be accurately identified in a timely manner, which will prevent disease progression and afford the best chance of an optimal outcome for fracture patients.

MicroRNAs (miRNAs/miRs), are short, noncoding single-stranded RNAs that regulate gene expression, and their roles as regulators of $\mathrm{BN}$ pathogenesis has been reported $(6,7)$. For example, one study found that miR-367-5p downregulation promoted the proliferation of osteoblasts in a model of microgravity-induced bone healing. These same researchers also determined that the gap junction structural protein pannexin-3 is a miR-367-5p target (8). Indeed, there is robust evidence that the expression of specific miRNAs is linked to the occurrence of BN (9-11). However, the exact molecular mechanisms by which miRNAs influence gene expression, and thereby regulate $\mathrm{BN}$ pathogenesis, remain incompletely understood, and as such, must be further studied in order to better prevent, treat and diagnose $\mathrm{BN}$.

Microarrays have been widely used to explore the pathogenic processes governing the development of a wide range of diseases, making them invaluable for functional genomic studies $(12,13)$. Several studies have employed microarrays to detect genes associated with BN-related processes, identifying a range of relevant proteins including lysyl oxidase like-2, chondroitin sulfate proteoglycan, aggrecan and collagen $\alpha-1$ (II) chain $(14,15)$. These differentially expressed genes (DEGs) are associated with the altered expression of proteins that have key structural and functional roles in this context, leading to their regulation of $\mathrm{BN}$ progression. 
Using microarray datasets analyzed via Gene Ontology (GO) and Kyoto Encyclopedia of Genes and Genomes (KEGG) pathway enrichment analyses, the present study sought to identify both DEGs and differentially expressed miRNAs (DEMs) associated with the progression of $\mathrm{BN}$.

\section{Materials and methods}

Data collection. The Gene Expression Omnibus (GEO) repository (National Center for Biotechnology Information) was reviewed to identify and obtain relevant datasets for the analysis of DEGs and DEMs. For DEGs, dataset GSE494 was downloaded, which was derived from the GPL8300 (HG_U95Av2) Affymetrix Human Genome U95 Version 2 Array platform. This dataset comprises four bone tissue samples, with two samples from BN patients and two from normal controls. Dataset GSE93390 was downloaded to identify DEMs, which was derived from the GPL14613 (miRNA-2) Affymetrix Multispecies miRNA-2 Array platform (16). This dataset contained miRNA expression levels for bone tissue samples from two BN patients and five normal controls.

Differential expression analysis. First, the Morpheus online tool (https://software.broadinstitute.org/morpheus/) was used to review the retrieved datasets, generating heat maps as a means of assessing overall changes in gene or miRNA expression and categorizing patients into $\mathrm{BN}$ and control groups. The GEO2R tool was subsequently used to identify DEGs and DEMs in patients with $\mathrm{BN}$, based on the following criteria: $\mathrm{P}<0.05$ and a fold-change of $\log ^{2} \geq 1$. Links to the GEO2R tools used for these analyses are as follows: https:/www.ncbi.nlm. nih.gov/geo/geo2r/?acc=GSE93390 and https://www.ncbi.nlm. nih.gov/geo/geo2r/?acc=GSE494

Protein-protein interaction (PPI) network and module analyses. The Search Tool for the Retrieval of Interacting Genes (v10.0; http://www.string-db.org/) was used to predict PPI pairs, followed by Cytoscape v3.7.1 (www.cytoscape. org/) to generate a PPI network for the identified DEGs. Node scores within this network were based on the degree of centrality, and nodes with higher scores were thus those more likely to be important within the PPI network, with the highest score indicating a hub protein within the network. Significant PPI network modules were then identified via the Molecular Complex Detection (MCODE) Cytoscape plugin (version 1.4.2; http://apps.cytoscape.org/apps/mcode), with a degree cutoff $\geq 2$ and k-core $\geq 3$.

Identification of DEM target genes. Next, miRDB (version 1.0; http://mirdb.org) was employed for DEM target gene prediction, followed once again by Cytoscape, to construct a DEM-target gene regulatory network. To identify points of overlap, the GSE494 DEGs were then overlaid with the target genes of the DEMs identified from GSE93390.

Functional enrichment analysis. Using the Database for Annotation, Visualization and Integrated Discovery tool v6.8 (http://david.abcc.ncifcrf.gov/), GO and KEGG pathway were both performed on all DEGs, module DEGs, and DEM target genes. $\mathrm{P}<0.05$ was the significance threshold.
Ethics approval. All experiments involving animals were conducted in compliance with the Guide for the Care and Use of Laboratory Animals by International Committees. The present study was approved by the Committees of Clinical Ethics in Tongji Medical College, Huazhong University of Science and Technology (Wuhan, China; 2016-049-83).

Femoral fracture models. A total of 20 male C57BL/6J mice (age, 8 weeks; weight, 20-25 g) were obtained from the Center of Experimental Animals (Tongji Medical College, Huazhong University of Science and Technology) in accordance with protocols approved by the Tongji Institutional Animal Care and Use Committee. Mice were single-caged and housed at room temperatures of $18^{\circ} \mathrm{C}$ with a $12 / 12 \mathrm{~h}$ light-dark cycle. The mice had free access to water and were fed a chow diet. Animals were anesthetized using an intraperitoneal injection (i.p.) of $10 \%$ chloral hydrate $(300 \mathrm{mg} / \mathrm{kg}$ body weight) was used for anesthesia via i.p., injection, after which no signs of peritonitis, pain or discomfort were observed. A longitudinal incision was created as in a previous study (17), and blunt separation of the underlying muscles (without removal of the periosteum) was performed to construct a mouse femoral fracture model. Transverse osteotomy of the femur was performed in the mid-diaphysis region using a diamond disk These fractures were then stabilized via a 23 -gauge intramedullary needle. On days 14 and 21 days post-surgery, the mice were anesthetized with an i.p. injection of $10 \%$ chloral hydrate $(300 \mathrm{mg} / \mathrm{kg}$ body weight), and then sacrificed by cervical dislocation, after which the callus was harvested for subsequent analysis.

Blood collection. From June 2016 to September 2018, peripheral blood samples from patients in Wuhan Union Hospital (6 healthy volunteers, 6 bone union patients and $6 \mathrm{BN}$ patients) were collected 1 day and 3 days post-surgery for determination of miRNA and mRNA levels. Patient characteristics are shown in Table SI. The patient studies were approved by the Committees of Clinical Ethics in the Union Hospital (Tongji Medical College, Huazhong University of Science and Technology), and informed consent was obtained from all participants.

Microcomputed tomography (mircoCT) analysis. The fracture site was scanned using the SkyScan 1276 scanner microCT system (Bruker Corporation) to provide images at 2,400 views, 5 frames/view, $37 \mathrm{kV}$, and $121 \mathrm{~mA}$, and these images were then analyzed with Bruker micro-CT evaluation software (Version 1.15.4.0; Bruker Corporation) to determine segmentation, three-dimensional morphometric analysis, density, and the following distance parameters: Bone volume (BV), tissue volume (TV), BV/TV and bone mineral density (BMD).

Cell culture and transfection. Human mesenchymal stem cells (hMSCs) were donated from the Orthopedic Laboratory of Tongji Medical College, Huazhong University of Science and Technology, and were cultured, in F12 media (Gibco; Thermo Fisher Scientific, Inc.) with 10\% fetal bovine serum (Gibco; Thermo Fisher Scientific, Inc.) and $1 \%$ penicillin/streptomycin (all Gibco; Thermo Fisher Scientific, Inc.). hMSCs were transfected with $20 \mu \mathrm{M}$ agomiR-193a-3p or antagomiR-193a-3p (Shanghai GenePharma Co., Ltd.) using Lipofectamine ${ }^{\circledR}$ 
3000 (Thermo Fisher Scientific, Inc.), according to manufacturer's protocol. Lipofectamine ${ }^{\circledR} 3000$ was also used to transfect cells with miRNAs or small interfering (si)RNA oligos. Mitogen-activated protein kinase 10 (MAPK10) siRNAs (Guangzhou RiboBio Co., Ltd.) were transfected at $50 \mathrm{nM}$. Then, $48 \mathrm{~h}$ after transfection, cells were collected for western blotting or reverse transcription-quantitative PCR (RT-qPCR). The sequences of siRNA MAPK10, agomiR- or anagomiR- were as follows: MAPK10 sense, 5'-CGCCAU CUAUGACAGUAAATT-3' and antisense, 5'-UUUACUGUC AUAGAUGGCGTT-3'; AntagomiR, 5'-ACUGGGACUUUG UAGGCCAGUU-3'; AgomiR-193a-3p sense, 5'-AACUGG CCUACAAAGUCCCAGU-3' and antisense strand, 5'-ACU GGGACUUUGUAGGCCAGUU-3'. AgomiR- was composed of double-stranded RNA with no chemical modifications. The 3 ' ends of the antagomiR and agomiR oligo nucleotides were conjugated to cholesterol, and all the bases were 2'-O methylated. The agomiRs, antagomiRs and siRNAs transfection kits (cat. nos. G04001, B05002 and B06002) were supplied by Shanghai GenePharma Co., Ltd.

$R T$ - $q P C R$. TRIzol $^{\circledR}$ reagent (Thermo Fisher Scientific, Inc.) was used to isolate total RNA from cell and tissue samples. The purified RNA was then reverse transcribed into cDNA using the ReverTra Ace ${ }^{\circledR}$ qPCR RT Master Mix (Toyobo Life Science), according to the manufacture's protocol. RT reaction was conducted for $15 \mathrm{~min}$ at $42^{\circ} \mathrm{C}$, followed by $5 \mathrm{~min}$ at $98^{\circ} \mathrm{C}$ and the reaction volume was $20 \mu \mathrm{l}$. The qPCR thermocycling conditions were: Intital denaturation at $95^{\circ} \mathrm{C}$ for $30 \mathrm{sec} ; 40$ cycles at $95^{\circ} \mathrm{C}$ for $5 \mathrm{sec}$ and $60^{\circ} \mathrm{C}$ for $30 \mathrm{sec}$, and the reaction volume was $25 \mu \mathrm{l}$. GAPDH served as an internal control. Relative miRNA expression levels were normalized to those of the internal control (GAPDH) and were calculated according to the $2^{-\Delta \Delta \mathrm{Cq}}$ method (18) All experiments were conducted in triplicate and the primer sequences are displayed in Table I.

Western blotting. The cells were washed three times with PBS three times and radio immunoprecipitation assay lysis buffer (Aspen Pharmacare Holdings Ltd.; cat. no. AS1004) was used to extract the total proteins from cells. Cell lysates $\left(1 \times 10^{4}\right)$ were subjected to $10 \%$ SDS-PAGE followed by determination of protein concentration by the bicinchoninic acid method. The proteins $(50 \mu \mathrm{g})$ were then transferred onto a $10 \%$ SDS-PVDF membrane. The PVDF membrane was blocked by $5 \%$ bovine serum albumin (Abcam) at room temperature for $2 \mathrm{~h}$. A chemiluminescence detection system (Canon, Inc.; cat. no. LiDE110) was then used to visualize proteins based on the provided instructions. Antibodies used were as follows: Anti-collagen I (1:500; Abcam; cat. no. ab34710), anti- alkaline phosphatase (ALP; 1:1,000; Abcam; cat. no. ab95462), anti-Osteocalcin (OCN; 1:500; Abcam; cat. no. ab93876), anti-Runt-related transcription factor 2 (Runx2; 1:500; Abcam; cat. no. ab23981) and anti-GAPDH (1:10,000; Abcam; cat. no. ab37168). All experiments were conducted in triplicate.

Luciferase reporter assay. The position 596-602 of 3'UTR of MAPK10 mRNA containing the putative target site of miR-193a-3p was determined by TargetScan (version 7.0; http://www.targetscan.org/vert_70/), and amplified by the same steps as mentioned above from the cDNA of hMSCs and ligated into the pGL3-basic vector (Promega Corporation). pGL3-MAPK10-3'UTR-mutant (Mut) was created by introducing two site mutations into miR-193a-3p potential target sites using Quick ChangeSite-Directed Mutagenesis kits (Agilent Technologies, Inc.). pGL3-MAPK10-3'UTR-wild-type (W; 200ng)orpGL3-MAPK10-3'UTR-Mut(200ng)was co-infected with Renilla plasmid into hMSCs using Lipofectamine ${ }^{\circledR} 3000$ (Thermo Fisher Scientific, Inc.). This was followed by transfection of miR-NC mimic (10 $\mathrm{nM})$ or miR-193a-3p mimic $(10 \mathrm{nM})$ for $48 \mathrm{~h}$ at $37^{\circ} \mathrm{C}$. The miR-NC mimic and miR-193a-3p mimic transfection kits (cat. nos. B05002 and B06002) were supplied by Shanghai GenePharma Co., Ltd. The sequence of miR-193a-3p mimic is as follow: sense, 5'-AACUGGCCU ACAAAGUCCCAGU-3' and antisense, 5'-ACUGGGACU UUGUAGGCCAGUU-3'. The Dual-Luciferase Reporter assay system (Promega Corporation) was used to measure the relative luciferase activity of each well. The firefly luciferase expression was normalized to Renilla.

Therapeutic stimulation with miR-193a-3p in fracture mice. A total of 20 mice were randomly divided into 3 groups, including a control group (injected locally with PBS), an agomiR-193a-3p group (injected with $100 \mu$ lagomiR-193a-3p) and an antagomiR-193a-3p group (injected with $100 \mu \mathrm{l}$ antagomiR-193a-3p). Each group was injected at the fracture site and injections were administered on days 1, 3, and 7 post-surgery.

Statistical analysis. GraphPad Prism 8.0 (GraphPad Software, Inc.) was used to conduct all analyses and the data are presented as the mean \pm standard deviation. The Student's t-test was used to compare two groups of data, whereas $\geq 3$ groups were compared using one-way analysis of variance with Tukey's post-hoc test. $\mathrm{P}<0.05$ was considered to indicate a statistically significant difference. All experiments were performed three times.

\section{Results}

$D E G$ identification. Across the two datasets analyzed in the present study, a total of $4 \mathrm{BN}$ patients and 7 control patient samples were analyzed. The Morpheus software was used to independently assess the gene expression profiles of these samples and the resultant DEG heat maps of the 30 top upregulated and downregulated genes in $\mathrm{BN}$ patients are shown in Fig. 1.

GO enrichment analysis. GO analysis revealed that the upregulated DEGs in patients with BN were associated with processes pertaining to the promotion of cell proliferation, cell responses to chemical stimuli and lymphocyte differentiation. By contrast, downregulated DEGs were associated with processes including 'phagocytosis', 'bacterial responses', 'responses to biotic stimuli', 'humoral immune responses' and 'extracellular matrix organization' (Table II).

KEGG pathway enrichment analysis. A KEGG pathway analysis revealed that the upregulated DEGs were those associated with cytokines, the toll-like receptor and TNF 
Table I. miRNAs and mRNA primer sequence.

\begin{tabular}{ll} 
miRNAs or gene name & \multicolumn{1}{c}{ Primer sequence $\left(5^{\prime} \rightarrow 3^{\prime}\right)$} \\
\hline $\begin{array}{l}\text { hsa-miR-193a-3p-Forward } \\
\text { hsa-miR-193a-3p-Reverse }\end{array}$ & ACACTCCAGCTGGGAACTGGCCTACAAAGT \\
H-miR-U6-Forward & TGGTGTCGTGGAGTCG \\
H-miR-U6-Reverse & CTCGCTTCGGCAGCACA \\
hsa-MAPK10-Forward & AACGCTTCACGAATTTGCGT \\
hsa-MAPK10-Reverse & CCAAGTATGCGGGACTCACCT \\
hsa-ALP-Forward & GGCTTGGCTGGCTTTGAGTT \\
hsa-ALP-Reverse & GCTCTGGAAAGTCCTTCAAAGC \\
hsa-COL1A1-Forward & TCTTCTTCCCTGGACACTGCC \\
hsa-COL1A1-Reverse & TGGCAAAGATGGACTCAACG \\
hsa-OCN-Forward & TCACGGTCACGAACCACATT \\
hsa-OCN-Reverse & TCACACTCCTCGCCCTATTG \\
hsa-Runx2-Forward & CTCCTGAAAGCCGATGTGGT \\
hsa-Runx2-Reverse & CTACTATGGCACTTCGTCAGGAT \\
H-GAPDH-Forward & ATCAGCGTCAACACCATCATT \\
H-GAPDH-Reverse & GGAAGCTTGTCATCAATGGAAATC
\end{tabular}

miR/miRNA, microRNA; ALP, alkaline phosphatase; hMSCs, Runx2, Runt-related transcription factor 2; Col1a1, collagen 1 $\alpha(\mathrm{I})$; NC, negative control; MAPK, mitogen associated protein kinase.

signaling pathways. By contrast, downregulated DEGs were primarily associated with pathways including 'ECM-receptor interaction', 'focal adhesion' and 'calcium signaling pathways' (Table III).

PPI network analysis. When the identified DEGs were analyzed, a total of 637 PPI pairs which were subsequently predicted to exist and these pairs were used in order to construct a PPI network; hub genes were then identified based on their location and degree of interaction within the network. Furthermore, 3 significant functional modules within this PPI network were further screened (Fig. 2). Enriched genes within module 1 included: CDKN3, RYR1, CXCL11, CCL2, KIAA0101, SAA1, CXCL2, IL1RN, IL6, CXCL8, CCL5, TTN, MYL1, MYH7, MYL3, IL1B, MYH2, TK1, TYMS, FOXM1, TNNI2, ACTA1, CXCL13, PTTG1, MYC, CKS2, MKI67, CCNB1, CDC20, TNNT3, TNNC2, CXCL3, FEN1, MYBPC1 and MYBPC2 (Table IV). Functional and pathway enrichment analyses of the genes were also performed (Table V).

Identification of DEMs. the GEO2R tool was used to identify DEMs in BN patients, based on the following criteria: $\mathrm{P}<0.05$ and a fold-change of $\log 2 \geq 1$. They were sorted in descending order of the absolute value of $\log F C$. A total of 20 DEMs, including top 10 that were upregulated (hsa-miR-129-5p, hsa-miR-1225-5p, hsa-miR-98, hsa-miR-149, hsa-miR-29b, hsa-miR-1263, hsa-miR-3185, hsa-miR-3128, hsa-miR-3187 and hsa-miR-126-5p) and top 10 that were downregulated (hsa-miR-199a-5p, hsa-miR-671-3p, hsa-miR-942, hsa-miR-335, hsa-miR-339-5p, hsa-miR-339-3p, hsa-miR-193a-3p, hsa-miR-504, hsa-miR-199b-5p and hsa-miR-345) were identified when $\mathrm{BN}$ tissue samples were compared with those of the controls (Table VI). Heat map analysis revealed clear differences in the pattern of miRNA expression patterns between patients with $\mathrm{BN}$ and the control group.

DEM target prediction and functional analysis. The miRDB database was used to identify 569 predicted DEM target genes and these target genes were then subjected to GO and KEGG analyses as conducted for the aforementioned DEGs (Table VII). The target genes were found to be significantly enriched for the following pathways and functions: 'Negative regulation of transcription by RNA polymerase II', 'positive regulation of transcription', 'DNA-templated', 'axon guidance', 'the estrogen signaling pathway', 'retrograde endocannabinoid signaling', and 'long-term potentiation'.

Integrated analysis of the DEG and DEM datasets. When the DEGs and DEM target genes were compared, a total of 9 common genes were identified between the two groups: ZBTB20, Cyclin L2 (CCNL2), PTPN9, ERCC1, CRLF1, SHH, PRCP, MAPK10, and MYH11 (Table VIII). However, only three of these showed the inverse regulatory relationships that one would expect for a miRNA-target gene pair: hsa-miR-1225-5p (upregulated)-CCNL2 (downregulated), hsa-miR-339-5p (downregulated)-PRCP (upregulated) and hsa-miR-193a-3p (downregulated)-MAPK10 (upregulated) (Table VII).

miR-193a-3p exerts a negative effect on osteoblast differentiation. As miR-193a-3p has been previously linked to cellular differentiation (17), the expression of miR-193a-3p in the serum of 18 patients in 3 groups (control, union and nonunion) was investigated by RT-qPCR. The results indicated that $72 \mathrm{~h}$ 
Table II. GO analysis of upregulated and downregulated differentially expressed genes in biological processes.

A, Upregulated

\begin{tabular}{llrr}
\hline Term & \multicolumn{1}{c}{ Function } & Count & P-value \\
\hline GO:0042127 & Regulation of cell population proliferation & 13 & $3.20 \times 10^{-6}$ \\
GO:0008284 & Positive regulation of cell population proliferation & 9 & $1.10 \times 10^{-6}$ \\
GO:0070887 & Cellular response to chemical stimulus & 16 & $1.40 \times 10^{-6}$ \\
GO:0008283 & Cell proliferation & 13 & $1.60 \times 10^{-5}$ \\
GO:0045621 & Positive regulation of lymphocyte differentiation & 4 & $1.10 \times 10^{-5}$ \\
\hline
\end{tabular}

B, Downregulated

\begin{tabular}{llrr}
\hline Term & \multicolumn{1}{c}{ Function } & Count & P-value \\
\hline GO:0006910 & Phagocytosis, recognition & 6 & $4.70 \times 10^{-7}$ \\
GO:0009617 & Response to bacterium & 14 & $8.30 \times 10^{-7}$ \\
GO:0009607 & Response to biotic stimulus & 17 & $1.30 \times 10^{-6}$ \\
GO:0006959 & Humoral immune response & 10 & $1.70 \times 10^{-6}$ \\
GO:0030198 & Extracellular matrix organization & 11 & $2.40 \times 10^{-6}$ \\
\hline
\end{tabular}

GO, Gene Ontology.

Table III. KEGG pathway analysis of upregulated and downregulated differentially expressed genes.

A, Upregulated

\begin{tabular}{lllll}
\hline Pathway ID & \multicolumn{1}{c}{ Name } & Count & P-value & \\
\hline hsa04060 & $\begin{array}{l}\text { Cytokine-cytokine receptor } \\
\text { interaction }\end{array}$ & 5 & $6.0 \times 10^{-4}$ & CCL20, CXCL11, CXCL8, MPL, OSM \\
hsa04620 & $\begin{array}{l}\text { Toll-like receptor signaling } \\
\text { pathway } \\
\text { TNFa04668 }\end{array}$ & 3 & $1.4 \times 10^{-4}$ & CXCL11, PTGS2, MAPK8 \\
\hline
\end{tabular}

B, Downregulated

\begin{tabular}{lllll}
\hline Pathway ID & \multicolumn{1}{c}{ Name } & Count & P-value & \\
\hline hsa04512 & ECM-receptor interaction & 6 & $6.9 \times 10^{-5}$ & CD47, COMP, CHAD, COL4A3, LAMC2, THBS4 \\
hsa04510 & Focal adhesion & 6 & $3.6 \times 10^{-3}$ & COMP, CHAD, COL4A3, LAMC2, MYLPF, THBS4 \\
hsa04020 & Calcium signaling pathway & 5 & $1.2 \times 10^{-2}$ & ATP2A1, CD38, GRM5, TRHR, TNNC2 KEGG \\
\hline
\end{tabular}

Kyoto Encyclopedia of Genes and Genomes.

post-injury, miR-193a-3p levels in BN group were increased compared with the other groups $72 \mathrm{~h}$ post-injury (Fig. 3A). Subsequently, the effect of miR-193a-3p on osteoblastogenesis was evaluated and the level of miR-193a-3p was found to be significantly upregulated in cells treated with agomiR-193a-3p (Fig. 3B and Fig. S1). Furthermore, the effects of miR-193a-3p were assessed in vitro, revealing a significant increase in bone formation markers in the antagomiR-193a-3p group (Fig. 3C and D).
miR-193a-3p directly targets MAPK10. Next, in order to investigate the downstream targets of miR-193a-3p, WT MAPK10 3'untranslated region (UTR) and Mut MAPK10 3'UTR constructs were cloned into luciferase reporters for use in a reporter assay, which revealed that agomiR-193a-3p, but not the agomiR-NC, significantly decreased WT MAPK10 3'UTR reporter activity (Fig. 4A). Moreover, to explore the association between miR-193a-3p and MAPK10, MAPK10 expression levels were measured during osteoblastic differentiation. In 


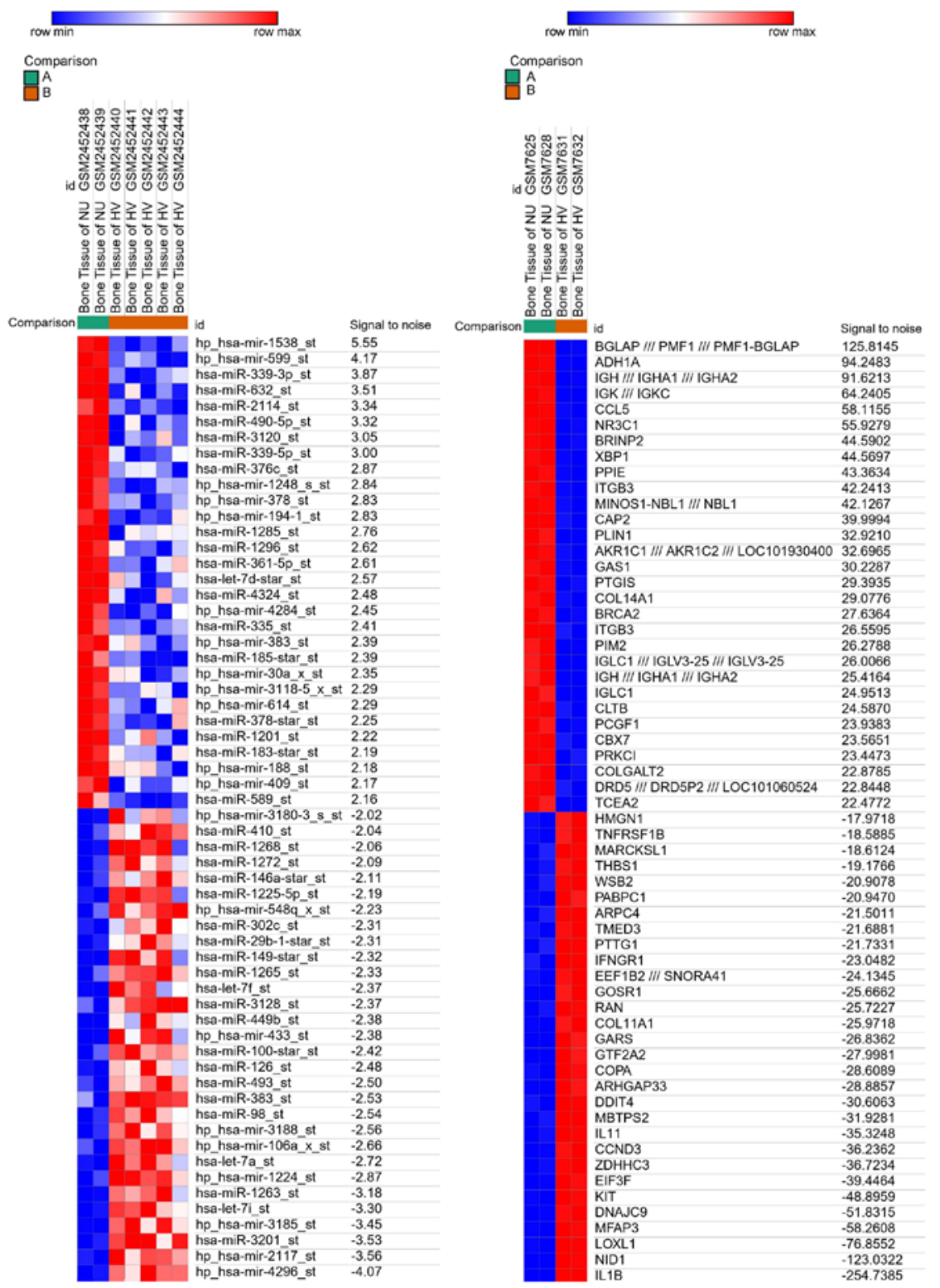

Figure 1. Heatmaps of DEGs and DEMs between BN patients and controls. BN patients and controls were completely separated by DEGs or DEMs. miRNAs, microRNAs, DEGs, differentially expressed genes; DEMs, differentially expressed miRNAs; BN, bone nonunion.

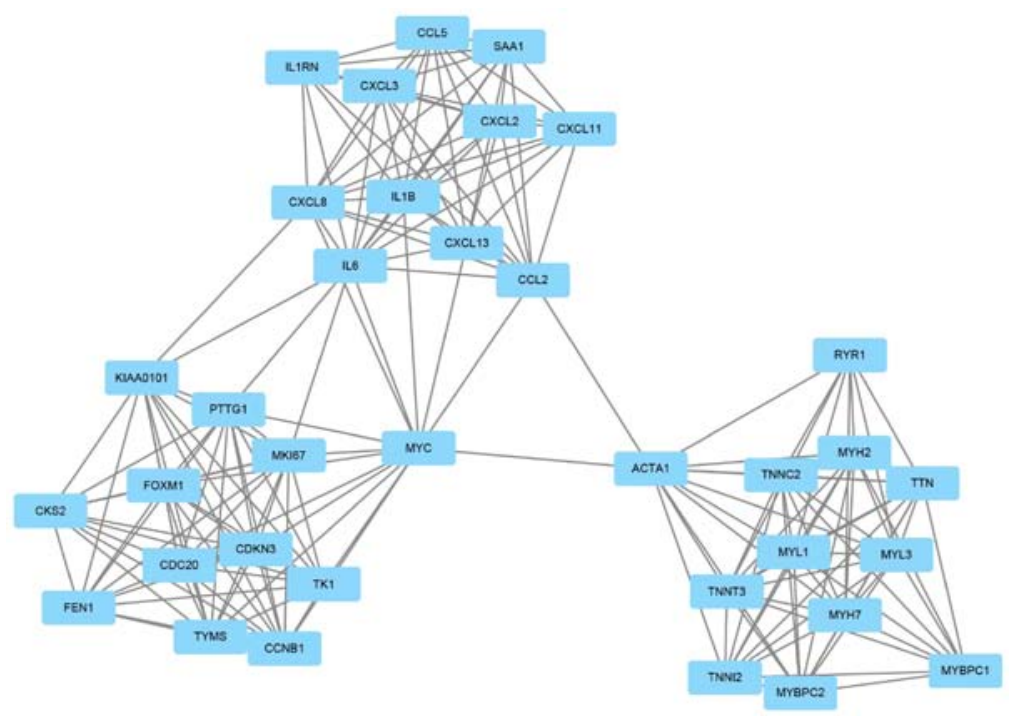

Figure 2. Top module from the protein-protein interaction network. 
Table IV. Three modules from the protein-protein interaction network satisfied the criteria of MCODE scores $\geq 4$ and number of nodes $>4$.

\begin{tabular}{|c|c|c|c|c|}
\hline Cluster & Score & Nodes & Edges & Node IDs \\
\hline 1 & 11.118 & 35 & 189 & $\begin{array}{l}\text { CDKN3, RYR1, CXCL11, CCL2, KIAA0101, SAA1, CXCL2, IL1RN, IL6, } \\
\text { CXCL8, CCL5, TTN, MYL1, MYH7, MYL3, IL1B, MYH2, TK1, TYMS, } \\
\text { FOXM1, TNN12, ACTA1, CXCL13, PTTG1, MYC, CKS2, MKI67, CCNB1, } \\
\text { CDC20, TNNT3, TNNC2, CXCL3, FEN1, MYBPC1, MYBPC2 }\end{array}$ \\
\hline 2 & 4.4 & 6 & 11 & CILP, COL2A1, COMP, PRG4, MATN4, COL11A1 \\
\hline 3 & 3 & 3 & 3 & HMOX1, FOS, SERPINE1 \\
\hline
\end{tabular}

Score $=($ Density $\mathrm{x}$ no. of node $)$.
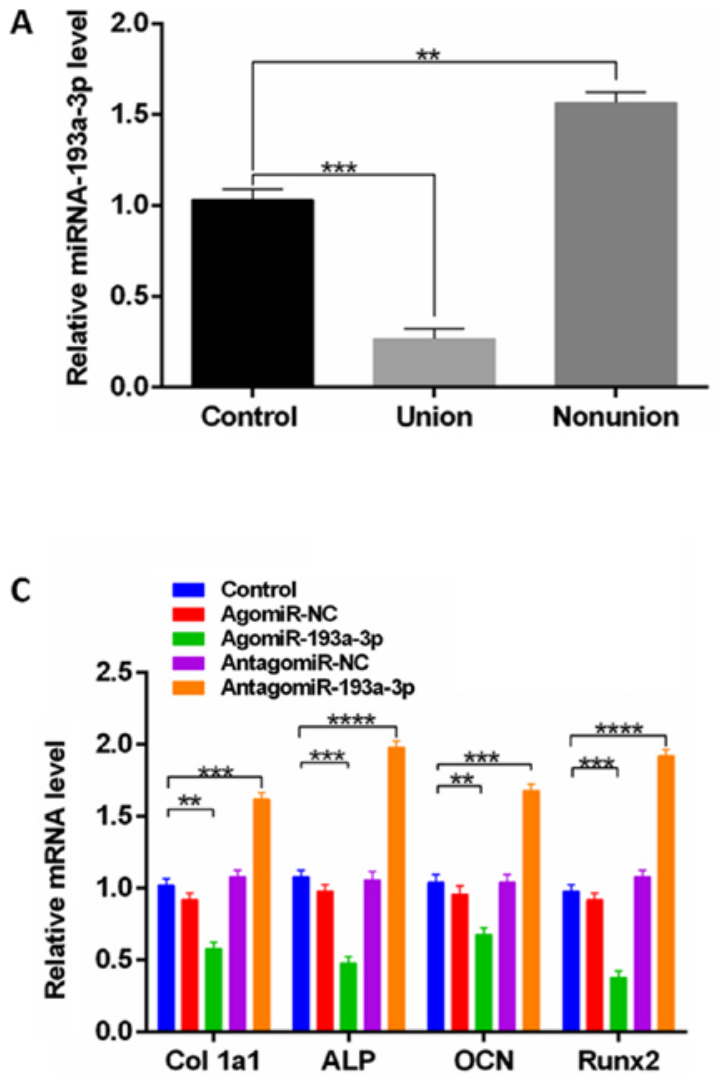

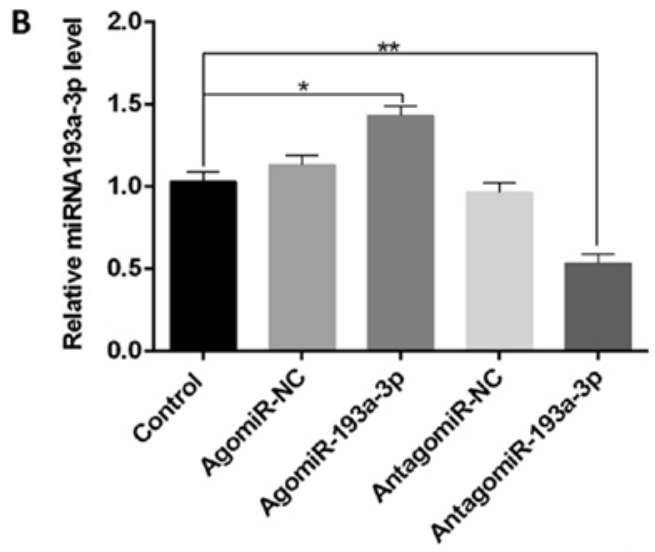

D

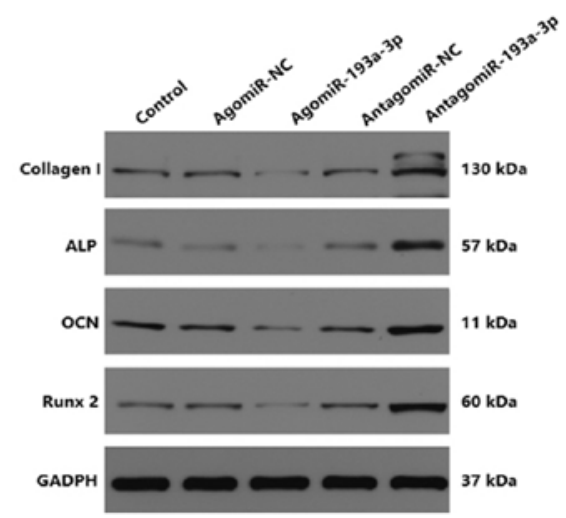

Figure 3. miR-193a-3p inhibits osteoblast activity. (A) Relative miR-193a-3p level was higher in nonunion group than other groups. (B) miR-193a-3p was upregulated in agomiR-193a-3p group following transfection for $48 \mathrm{~h}$. (C) PCR and western blotting (D) of ALP, Colla1, OCN and Runx2 protein levels in hMSCs cells treated with transfection constructs for $48 \mathrm{~h}$. Data are means \pm standard deviation of triplicate experiments. ${ }^{*} \mathrm{P}<0.05,{ }^{* *} \mathrm{P}<0.01$ and ${ }^{* * * *} \mathrm{P}<0.001$. miR, microRNA; ALP, alkaline phosphatase; hMSCs, human mesenchymal stem cells; Runx2, Runt-related transcription factor 2; Col1a1, collagen 1 $\alpha(\mathrm{I})$; NC, negative control.

addition, serum samples were collected from control, union or nonunion patients and they were analyzed via RT-qPCR, which revealed clear MAPK10 downregulation in nonunion patients relative to other groups (Fig. 4B). In vitro, lower relative MAPK10 mRNA levels in the agomiR-193a-3p group were observed compared with other groups (Fig. 4C). Furthermore, to test whether osteoblast differentiation was MAPK10-dependent, the effect of a MAPK10-specific siRNA on osteoblastogenesis was evaluated. RT-qPCR and western blotting analysis indicated that siRNA-MAPK10 downregulated Col1-a1, ALP, OCN, and Runx2 (Fig. 4D and E).
Local injection of miR-193a-3p inhibits fracture healing in mice. Finally, PBS, agomiR-193a-3p, or antagomiR-193a-3p were directly injected into the local fracture sites of model animals to assess whether agomiR-193a-3p was able to improve fracture healing. Additionally, RT-qPCR analysis was performed for the mice bone tissues to testify the overexpression/knockdown of miR-193a-3p level (Fig. S2). Local injection was performed at three time points on days 1 , 3 and 7 post fracture respectively, and microCT examination was performed on days 14 and 21 post-fracture. The results indicated a smaller total and bone callus volume in 


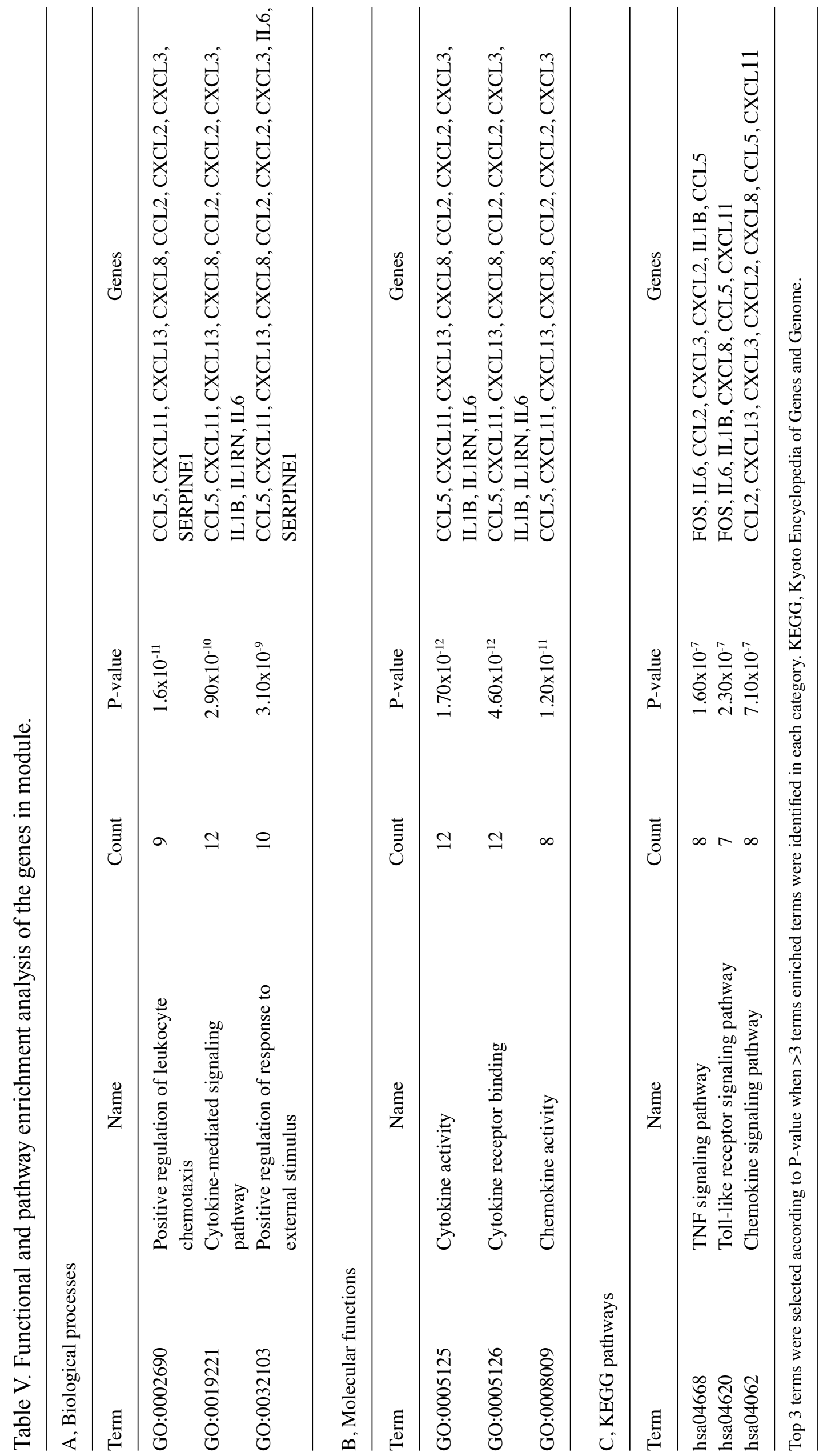


Table VI. Differentially expressed miRNAs between nonunion patients and healthy volunteers.

\begin{tabular}{lcc}
\hline miRNA & logFC & P-value \\
\hline hsa-miR-129-5p & 2.08 & $1.06 \times 10^{-3}$ \\
hsa-miR-1225-5p & 1.86 & $2.25 \times 10^{-3}$ \\
hsa-miR-98 & 1.79 & $3.53 \times 10^{-4}$ \\
hsa-miR-126-5p & 1.72 & $1.20 \times 10^{-3}$ \\
hsa-miR-149-star & 1.40 & $3.37 \times 10^{-3}$ \\
hsa-miR-29b-1-star & 1.37 & $1.50 \times 10^{-3}$ \\
hsa-miR-1263 & 1.27 & $6.88 \times 10^{-4}$ \\
hsa-mir-3185 & 1.15 & $6.19 \times 10^{-4}$ \\
hsa-miR-3128 & 1.13 & $3.48 \times 10^{-4}$ \\
hsa-miR-3187 & 1.03 & $3.04 \times 10^{-3}$ \\
hsa-miR-199a-5p & -2.28 & $2.96 \times 10^{-3}$ \\
hsa-miR-671-3p & -1.71 & $1.11 \times 10^{-3}$ \\
hsa-miR-942 & -1.70 & $1.31 \times 10^{-3}$ \\
hsa-miR-335 & -1.60 & $1.08 \times 10^{-4}$ \\
hsa-miR-339-5p & -1.47 & $4.68 \times 10^{-4}$ \\
hsa-miR-339-3p & -1.28 & $2.60 \times 10^{-4}$ \\
hsa-miR-193a-3p & -1.19 & $9.31 \times 10^{-4}$ \\
hsa-miR-504 & -1.15 & $3.92 \times 10^{-4}$ \\
hsa-miR-199b-5p & -1.14 & $2.87 \times 10^{-3}$ \\
hsa-miR-345 & -1.13 & $3.46 \times 10^{-3}$ \\
\hline
\end{tabular}

FC, fold-change; miRNA, microRNA.

agomiR-193a-3p animals compared with the control and anta gomiR-193a-3p-treated-animals (Fig. 5A-C). In addition, agomiR-193a-3p-treated-animals exhibited lower BMD than did animals in the other two groups (Fig. 5D). In summary, these results indicated that miRNA-193a-3p plays a negative role in fracture healing.

\section{Discussion}

In the present study, mRNA and miRNA expression datasets from $\mathrm{BN}$ and control patients were integrated, allowing the identification of hsa-miR-1225-5p, hsa-miR-339-5p and hsa-miR-193-3p, and their respective target genes CCNL2, PRCP, and MAPK10 as potential biomarkers useful for BN diagnosis. Based on functional enrichment analyses and previous publications, it was also further determined that CCNL2 may regulate BN development via the estrogen signaling pathway, whereas PRCP and MAPK10 may do so via retrograde endocannabinoid signaling.

The estrogen signaling pathway plays a key role in regulating the functionality and proliferation of cells in numerous contexts (19-22). Endometrial cell proliferation is regulated via the estrogen-induced brain-derived neurotrophic factor signaling pathway $(23,24)$. It has also been shown that $17 \beta$ estradiol is able to prevent bone deterioration at least in part via suppressing ephA2/ephrinA2 signaling $(25,26)$. As such, the regulation of estrogen signaling has been explored as a potential treatment for promoting bone formation. For example, a previous study found that 8-O-4'norlignan was able to activate an endoplasmic reticulum signaling pathway in osteoblast-like cells in a ligand-independent, estrogen response element-independent and MAPK-dependent manner (27). Another study determined that estrogen receptor- $\alpha$ was expressed in both osteoblasts and osteoclasts, and they further found that estrogen-mediated activation of this receptor within osteoblast progenitor cells promoted cortical bone accrual (28). Transforming growth factor $\beta$-inducible early gene- 1 is also known to regulate estrogen signaling in bone tissue (29). In the present study, it was found that ZBTB20, PTPN9 and ERCC1 were all upregulated in individuals with $\mathrm{BN}$, confirming the relevance of estrogen-associated proliferation and apoptosis in BN.

Previous research suggests that CCNL2 can negatively regulate blood vessel remodeling (30). Further studies have also found that this gene also enhances intracellular intron splicing activity in a manner opposed by the expression of cyclin dependent kinase (CDK)11 (p58/p46) or inactive CDK11 (p110), resulting in differential mRNA splicing as a function of CCNL2 expression (31-33). A previous study suggested CCNL2 to be a cyclin family gene member that has the potential to regulate the transcription and subsequent RNA processing for genes which regulate apoptosis, thereby impairing cellular proliferation and potentially promoting apoptotic cell death (31). In the present study, CCNL2 was found to be upregulated in samples from patients with $\mathrm{BN}$, wherein it has the potential to cause osteoblast apoptosis. At present to the best of our knowledge, no previous studies have examined the role of CCNL2 in BN and as such, further studies are warranted.

The current study revealed that PRCP and MAPK10 are involved in the retrograde endocannabinoid signaling pathway, which has the potential to activate presynaptic type 1 cannabinoid receptors to depress neurotransmitter release. As such, it is speculated that PRCP and MAPK10 may regulate BN by mediating the release of special neurotransmitters, which in turn may regulate bone remodeling $(33,34)$. Indeed, synaptic function is known to be regulated by endocannabinoids, which are lipid signaling molecules that regulate signaling within the central nervous system (35). This may explain the downregulation of PRCP and MAPK10 in the BN patients of the present study.

miRNAs have previously attracted attention as potential biomarkers of BN (36-39), warranting the identification of DEMs in BN patient bone tissue samples. The present study revealed that miR-1225-5p is highly expressed in BN tissues. The upregulation of miR-1225-5p has also been associated with the impaired proliferation of laryngeal carcinoma cells as a result of G1/S phase cell cycle arrest. By contrast, reduced miR-1225-5p expression was associated with increased cell survival (40). In line with this, miR-1225-5p was found to regulate CCNL2 potentially promoting cellular proliferation and inhibiting apoptotic cell death, though further research is required to experimentally validate the relationship between miR-1225-5p and CCNL2.

Elevated plasma miR-339-5p levels have previously been identified as a biomarker of lung adenocarcinoma (39) and linked to increased activation of the protein B-cell lymphoma 6 (41), whereas upregulation of the resulting protein suppresses PRCP expression $(42,43)$. Consistent with these findings, the present study revealed that miR-339-5p was upregulated in 

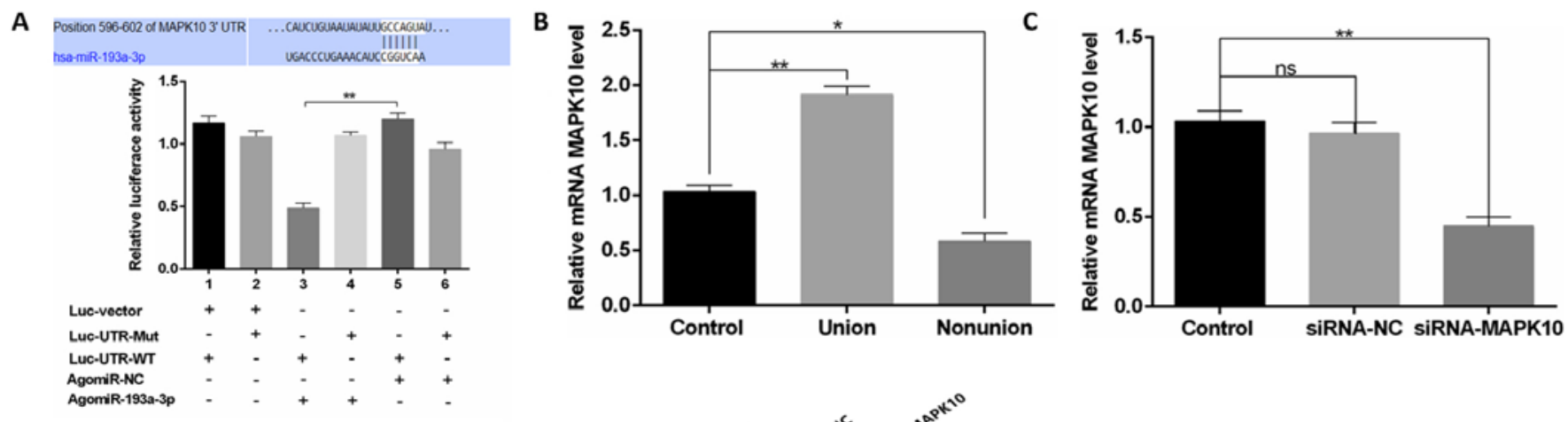

D

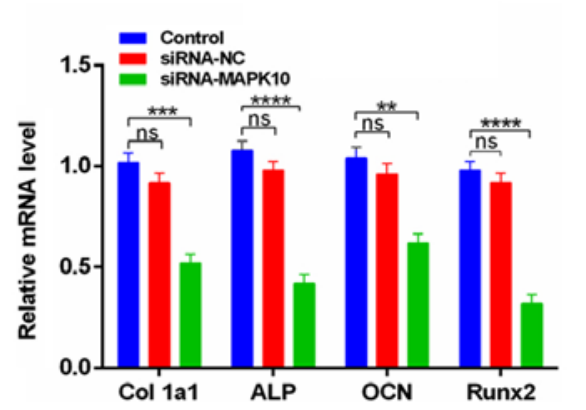

E

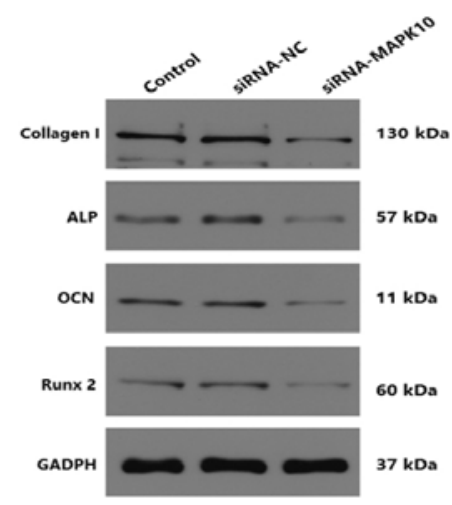

Figure 4. miR-193a-3p targets MAPK10 to inhibit osteoblast activity in vitro. (A) miR-193a-3p effects in hMSCs cells on luciferase activity following antagomiR-NC or antagomiR-193a-3p treatment. (B) Relative MAPK10 level was lower in the nonunion group than other groups. (C) Relative MAPK10 level was lower in agomiR-193a-3p group than other groups. (D) PCR and (E) western blotting analysis were used following transfection to assess Colla1, ALP, OCN, and Runx2 expression. Data are the mean \pm standard deviation of triplicate experiments. ${ }^{*} \mathrm{P}<0.05,{ }^{* * *} \mathrm{P}<0.01,{ }^{* * * *} \mathrm{P}<0.001,{ }^{* * * *} \mathrm{P}<0.0001$. miR, microRNA; ALP, alkaline phosphatase; hMSCs, human mesenchymal stem cells; Runx2, Runt-related transcription factor 2; Colla1, collagen 1 $\alpha(\mathrm{I})$; NC, negative control; MAPK, mitogen associated protein kinase; ns, not significant; Mut, mutant; WT, wild type; UTR, untranslated region.
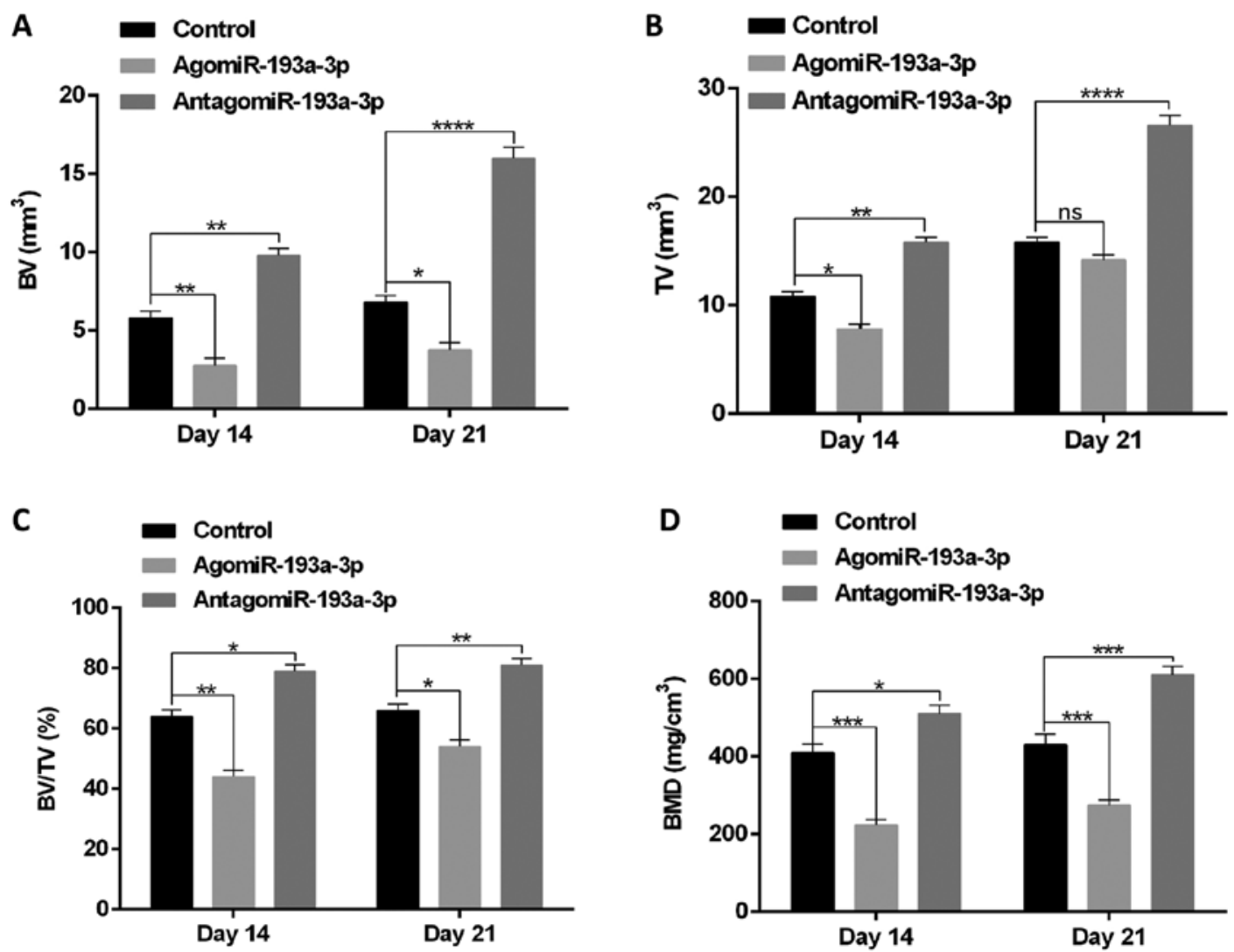

Figure 5. Local injection of agomiR-193a-3p inhibits fracture healing in mice. PBS, agomiR-193a-3p and antagomiR-193a-3p were injected locally to the fracture site on days 1,3 , and 7 post-fracture. BV (A) and TV (B) of the callus, BV/TV (C) and BMD (D) on days 14 and 21 post-operation were established via microcomputed tomography. $\mathrm{n}=10$ mice/group. Data are means \pm standard deviation of triplicate experiments. ${ }^{*} \mathrm{P}<0.05,{ }^{* *} \mathrm{P}<0.01,{ }^{* * *} \mathrm{P}<0.001,{ }^{* * * *} \mathrm{P}<0.0001 . \mathrm{BV}$, bone volume; TV, tissue volume; BMD, bone mineral density; miR, microRNA; ns, not significant. 
Table VII. GO and KEGG pathways enrichment for target genes of differentially expressed miRNAs.

A, Biological processes

\begin{tabular}{|c|c|c|c|c|}
\hline Term & Name & Count & P-value & Genes \\
\hline GO:0000122 & $\begin{array}{l}\text { Negative regulation } \\
\text { of transcription by } \\
\text { RNA polymerase II }\end{array}$ & 93 & $2.1 \times 10^{-10}$ & $\begin{array}{l}\text { ARID5B, BCL6, BCOR, BEND3, BACH2, CTBP2, } \\
\text { CGGBP1, CREBRF, DAB2IP, DCAF1, DNMT3A, } \\
\text { DNAJB5, EP300, ELK4, ETV3L, ETV6, JUNB, KLF11, } \\
\text { KLF17, MAF, MXD4, MDM2, MDM4, MLXIPL, MLX, } \\
\text { NIPBL, OTUD7B, PHF12, PHF21A, PRDM1, PRDM5, } \\
\text { RB1, REL, SATB1, SMAD4, SP100, SOX11, SOX6, SP3, } \\
\text { TAL1, TGIF2, WWC1, WWTR1, ZFP90, ATF7IP, BPTF, } \\
\text { CBX4, CBX6, CBX7, CIITA, CUX1, CDKN1C, CPEB3, } \\
\text { ESR1, FNIP2, FLCN, FST, FOXP2, HSBP1, HMGA2, } \\
\text { HMGB1, HDAC9, HIPK2, JARID2, KDM5A, MTDH, } \\
\text { MECP2, MEF2A, NFIB, NFIC, NFKB1, NR4A2, NFX1, } \\
\text { PAX6, PIAS4, SIM2, SHH, TSHZ1, TSHZ2, THRB, } \\
\text { TCF4, TBL1XR1, TRIM33, USP9X, VEGFA, ZEB2, } \\
\text { ZBTB20, ZBTB4, ZBTB7A, ZFHX3, ZNF280D, ZNF281 }\end{array}$ \\
\hline GO:0045893 & $\begin{array}{l}\text { Positive regulation } \\
\text { of transcription, } \\
\text { DNA-templated }\end{array}$ & 67 & $7.6 \times 10^{-8}$ & $\begin{array}{l}\text { CTCFL, CNBP, DAB2, ELK3, ETS1, KLF6, KLF7, LHX2, } \\
\text { MLXIPL, NAA15, NIF3L1, POU3F1, PIM2, RORA, RB1, } \\
\text { SMAD4, SP100, SOX11, SOX4, SP3, TAL1, WNT5A, } \\
\text { ZFP90, ATF5, ATF7IP, AR, BPTF, CREB5, CAMK4, } \\
\text { CIITA, CLOCK, F2R, COL1A1, CDKN1C, ERBB4, } \\
\text { ESR1, FOXN3, FZD4, GRIP1, HMGA2, HIPK2, IGF1, } \\
\text { IRF1, LBH, KAT6B, KDM5A, KDM7A, KMT2A, } \\
\text { MECP2, MAPK1, NFKB1, NFATC3, NCOA1, PAX6, } \\
\text { PHIP, RFX3, RET, RUNX1, SHH, TCF4, TFAP4, } \\
\text { TBL1XR1, ZFHX3, ZNF281, ZNF516, ZXDA }\end{array}$ \\
\hline GO:0007411 & Axon guidance & 31 & $1.0 \times 10^{-7}$ & $\begin{array}{l}\text { EPHA8, EPHB2, EPHB3, ETV1, KLF7, L1CAM, } \\
\text { LHX2, SMAD4, SOS1, WNT5A, APBB2, ANK3, BDNF, } \\
\text { CNTN4, ENAH, KIF26A, MATN2, MAPK1, NTN1, } \\
\text { NRXN1, NRXN3, NFASC, PAX6, RPS6KA5, SEMA3A, } \\
\text { EMA6A, SIAH1, SHH, UNC5C, UNC5D, ZNF280D }\end{array}$ \\
\hline
\end{tabular}

B, KEGG pathways

\begin{tabular}{|c|c|c|c|c|}
\hline Term & Name & Count & P-value & Genes \\
\hline hsa04915 & $\begin{array}{l}\text { Estrogen signaling } \\
\text { pathway }\end{array}$ & 20 & $8.2 \times 10^{-6}$ & $\begin{array}{l}\text { ADCY1, ADCY2, CCNL2, FKBP5, ESR1, CREB5, } \\
\text { GABBR2, GRM1, ITPR1, ITPR2, MAPK1, GNAQ, } \\
\text { SOS1, SOS2, SHC1, PRKACB, PLCB2, PIK3R1, } \\
\text { CALM1, SHC4 }\end{array}$ \\
\hline hsa04723 & $\begin{array}{l}\text { Retrograde endocannabinoid } \\
\text { signaling }\end{array}$ & 20 & $1.1 \times 10^{-5}$ & $\begin{array}{l}\text { GABRG1, ADCY1, ADCY2, GNAI3, GABRA4, } \\
\text { GABRA3, GABRB1, GRIA4, GRM1, ITPR1, PRCP, } \\
\text { ITPR2, GRM5, MAPK10, SLC17A6, GNAQ, MGLL, } \\
\text { PRKACB, PLCB2, CACNA1B }\end{array}$ \\
\hline hsa04720 & Long-term potentiation & 15 & $4.0 \times 10^{-5}$ & $\begin{array}{l}\text { ADCY1, GRIN2A, GRM1, ITPR1, PRKCB, ITPR2, } \\
\text { GRM5, MAPK1, EP300, CAMK4, GNAQ, CAMK2D, } \\
\text { PRKACB, PLCB2, CALM1 }\end{array}$ \\
\hline
\end{tabular}

KEGG, Kyoto Encyclopedia of Genes and Genome; GO, Gene Ontology.

the BN samples, with the potential to negatively regulate its target gene, PRCP. However, further experimental validation of the relationship between miR-339-5p and PRCP expression is required. 
Table VIII. The genes of bone tissue and their regulatory miRNAs.

\begin{tabular}{ll} 
miRNA name & Gene symbol \\
\hline hsa-miR-129-5p & ZBTB20 \\
hsa-miR-1225-5p & CCNL2 \\
hsa-miR-126-5p & PTPN9 \\
hsa-mir-3185 & ERCC1 \\
hsa-miR-199a-5p & CRLF1 \\
hsa-miR-671-3p & SHH \\
hsa-miR-339-5p & PRCP \\
hsa-miR-193a-3p & MAPK10 \\
hsa-miR-199b-5p & MYH11
\end{tabular}

miR/miRNA, microRNA.

It is also known that miR-193a-3p serves key roles in regulating the metastasis of osteosarcoma by downregulating both the Rab27B and serine racemase genes, and as such, miR-193a-3p has been proposed as a biomarker of metastatic osteosarcoma (44). Advanced osteosarcoma is also associated with increased bone damage and osteoclast activation, and therefore, miR-193a-3p may also be downregulated in $\mathrm{BN}$, as was observed in the present study. To the best of our knowledge, no previous studies have documented a negative association between miR-193a-3p and MAPK10 in the context of BN, and as such this was a novel finding of the present study. These results also illustrated that the expression of miR-193a-3p was increased in patients with $\mathrm{BN}$ compared with those with bone union and the negative effect of miR-193a-3p on hMSC osteoblastic differentiation was demonstrated in vitro. In addition, a fracture mouse model was used to assess the effect of miR-193a-3p on fracture healing in vivo. Collectively, these results helped to support the in vitro and in silico findings of the present group.

To conclude, the present study revealed that miR-193a-3p and its target gene MAPK10 may be a potential biomarkers for $\mathrm{BN}$, although further investigation with a larger sample size is required to validate their potential values as future diagnostic, prognostic and/or therapeutic biomarkers. However, there still exits some limitations in the present study. Firstly, as it appears to be common practice to adjust $\mathrm{P}$-values for multiple testing during bioinformatics analysis and adjusted P-values in the authors' subsequent studies will be applied. Moreover, bioinformatics analysis and multiple experimental tools were used to screen for potential key markers of BN. However, sensitivity and specificity of this potential biomarkers had not been explored in the current study, and the present group aims to highlight these points in their future research. Additionally, the small sample size and types of patient was another limitation of the present study.

\section{Acknowledgements}

Not applicable.

\section{Funding}

This study was supported by the National Science Foundation of China (grant no. 81772345), the Healthy Commission Key Project of Hubei Province (grant no. WJ2019Z009), the Science and Technology Department of Hubei Province (grant no. 2016CFB424), the Development Center for Medical Science and Technology National Health and Family Planning Commission of the People's Republic of China (ZX-01-C2016024).

\section{Availability of data and materials}

The datasets used and/or analyzed during the current study are available from the corresponding author on reasonable request.

\section{Authors' contributions}

YX and LC designed the study. FC and XL performed data curation. YC and YX carried out the statistical analysis. GL and $\mathrm{CY}$ performed the investigations. $\mathrm{BM}$ and $\mathrm{WZ}$ were responsible for project administration. YE and GL operated the software. YE was responsible for supervision. BM and WZ performed validation. CY and YC conceived the study, participated in its design and coordination and helped to draft the manuscript. GL, YX, and WZ participated in the sequence alignment and drafted the manuscript. All authors read and approved the final manuscript.

\section{Ethics approval and consent to participate}

All experiments involving animals were conducted in compliance with the Guide for the Care and Use of Laboratory Animals by International Committees. The present study was approved by the Committees of Clinical Ethics in Tongji Medical College, Huazhong University of Science and Technology (Wuhan, China) approved this study (2016-049-83). The patient studies were approved by the Committees of Clinical Ethics in the Union Hospital (Tongji Medical College, Huazhong University of Science and Technology, Wuhan, China), and informed consent was obtained from all participants.

\section{Patient consent for publication}

Not applicable.

\section{Competing interests}

The authors declare that they have no competing interests.

\section{References}

1. DiSilvio F Jr, Foyil S, Schiffman B, Bernstein M, Summers H and Lack WD: Long bone union accurately predicted by cortical bridging within 4 months. JB JS Open Access 3: e12, 2018.

2. Supakul S, Yao K, Ochi H, Shimada T, Hashimoto K, Sunamura S, Mabuchi Y, Tanaka M, Akazawa C, Nakamura T, et al: Pericytes as a source of osteogenic cells in bone fracture healing. Int J Mol Sci 20: E1079, 2019.

3. Hovius SE and de Jong T: Bone grafts for scaphoid nonunion: An overview. Hand Surg 20: 222-227, 2015. 
4. Zaidenberg EE, Juarez Cesca F, Pastrana MJ and Zaidenberg CR Pedicled vascularized bone graft of the distal radius for recalcitrant nonunion of the distal humerus. J Orthop Trauma 32: e394-e399, 2018

5. Attanayake AMHS, De Silva UMGD, Jayaweera JAAS and Perera DL: Use of Ayurveda and Sri Lankan traditional medicine for healing shaft of humerus fracture following nonunion. J Ayurveda Integr Med 9: 217-220, 2018.

6. Zheng W and Guan J: Oncostatin M promotes the osteogenic differentiation of mouse MC3T3-Elosteoblasts through the regulation of monocyte chemotactic protein-1. Mol Med Rep 18: 2523-2530, 2018.

7. Zhang P, Gao H, Li Q, Chen X and Wu X: Downregulation of microRNA-660 inhibits cell proliferation and invasion in osteosarcoma by directly targeting forkhead box O1. Mol Med Rep 18: 2433-2440, 2018.

8. Jia HL and Zhou DS: Downregulation of microRNA-367 promotes osteoblasts growth and proliferation of mice during fracture by activating the PANX3-mediated Wnt/ $\beta$-catenin pathway. J Cell Biochem 2018 (Epub ahead of print).

9. Frohlich LF: Micrornas at the interface between osteogenesis and angiogenesis as targets for bone regeneration. Cells 8: E121, 2019.

10. Zu H, Yi X and Zhao D: Transcriptome sequencing analysis reveals the effect of combinative treatment with low-intensity pulsed ultrasound and magnesium ions on hFOB1.19 human osteoblast cells. Mol Med Rep 18: 749-762, 2018.

11. Mao W andZhuZ: Parthenolide inhibits hydrogen peroxide-induced osteoblast apoptosis. Mol Med Rep 17: 8369-8376, 2018.

12. Kang S and Song J: Robust gene selection methods using weighting schemes for microarray data analysis. BMC Bioinformatics 18: 389, 2017.

13. Lin SM, Du P, Huber W and Kibbe WA: Model-based variance-stabilizing transformation for Illumina microarray data. Nucleic Acids Res 36: e11, 2008.

14. Wang A, Ren M, Song Y, Wang X, Wang Q, Yang Q, Liu H Du Z, Zhang G and Wang J: MicroRNA expression profiling of bone marrow mesenchymal stem cells in steroid-induced osteonecrosis of the femoral head associated with osteogenesis. Med Sci Monit 24: 1813-1825, 2018.

15. Waki T, Lee SY, Niikura T, Iwakura T, Dogaki Y, Okumachi E, Oe K, Kuroda R and Kurosaka M: Profiling microRNA expression during fracture healing. BMC Musculoskelet Disord 17: 83, 2016.

16. Fortriede JD, Pells TJ, Chu S, Chaturvedi P, Wang D, Fisher ME James-Zorn C, Wang Y, Nenni MJ, Burns KA, et al: Xenbase: Deep integration of GEO \& SRA RNA-seq and ChIP-seq data in a model organism database. Nucleic Acids Res gkz933, 2019 (Epub ahead of print).

17. Xiong Y, Cao F, Hu L, Yan C, Chen L, Panayi AC, Sun Y, Zhou W, Zhang P and Wu Q: miRNA-26a-5p accelerates healing via downregulation of PTEN in fracture patients with traumatic brain injury. Mol Ther Nucleic Acids 17: 223-234, 2019.

18. Livak KJ and Schmittgen TD: Analysis of relative gene expression data using real-time quantitative PCR and the 2(-Delta Delta C(T)) method. Methods 25: 402-408, 2001.

19. Wang W, Chen J, Hui Y, Huang M and Yuan P: Down-regulation of miR-193a-3p promotes osteoblast differentiation through up-regulation of LGR4/ATF4 signaling. Biochem Biophys Res Commun 503: 2186-2193, 2018.

20. Zhu Y, Kawaguchi K and Kiyama R: Differential and directional estrogenic signaling pathways induced by enterolignans and their precursors. PLoS One 12: e171390, 2017.

21. Vrtacnik P, Ostanek B, Mencej-Bedrac S and Marc J: The many faces of estrogen signaling. Biochem Med (Zagreb) 24: 329-342, 2014.

22. Kiyama R: Estrogenic terpenes and terpenoids: Pathways, functions and applications. Eur J Pharmacol 815: 405-415, 2017.

23. Dong F, Zhang Q, Kong W, Chen J, Ma J, Wang L, Wang Y, Liu Y, Li Y and Wen J: Regulation of endometrial cell proliferation by estrogen-induced BDNF signaling pathway. Gynecol Endocrinol 33: 485-489, 2017.

24. Crider A and Pillai A: Estrogen signaling as a therapeutic target in neurodevelopmental disorders. J Pharmacol Exp Ther 360: $48-58,2017$.

25. Liu L, Zhou L, Yang X, Liu Q, Yang L, Zheng C, Zhao Y, Zhang Z and Luo X: 17ß-estradiol attenuates ovariectomy induced bone deterioration through the suppression of the ephA2/ephrinA2 signaling pathway. Mol Med Rep 17: 1609-1616, 2018.

26. Yin X, Wang X, Hu X, Chen Y,Zeng K and Zhang H: ER $\beta$ induces the differentiation of cultured osteoblasts by both Wnt/B-catenin signaling pathway and estrogen signaling pathways. Exp Cell Res 335: 107-114, 2015.
27. Xiao HH, Gao QG, Ho MX, Zhang Y, Wong KC, Dai Y, Yao XS and Wong MS: An 8-O-4' norlignan exerts oestrogen-like actions in osteoblastic cells via rapid nongenomic ER signaling pathway. J Ethnopharmacol 170: 39-49, 2015

28. Almeida M, Iyer S, Martin-Millan M, Bartell SM, Han L, Ambrogini E, Onal M, Xiong J, Weinstein RS, Jilka RL, et al: Estrogen receptor- $\alpha$ signaling in osteoblast progenitors stimulates cortical bone accrual. J Clin Invest 123: 394-404, 2013.

29. Hawse JR, Pitel KS, Cicek M, Philbrick KA, Gingery A, Peters KD, Syed FA, Ingle JN, Suman VJ, Iwaniec UT, et al: TGF $\beta$ inducible early gene- 1 plays an important role in mediating estrogen signaling in the skeleton. J Bone Miner Res 29: 1206-1216, 2014.

30. Liu H, Tao Y, Chen M, Yu J, Li WJ, Tao L, Li Y and Li F: Upregulation of MicroRNA-214 contributes to the development of vascular remodeling in hypoxia-induced pulmonary hypertension via targeting CCNL2. Sci Rep 6: 24661, 2016.

31. Loyer P, Trembley JH, Grenet JA, Busson A, Corlu A, Zhao W, Kocak M, Kidd VJ and Lahti JM: Characterization of cyclin L1 and L2 interactions with CDK11 and splicing factors: Influence of cyclin L isoforms on splice site selection. J Biol Chem 283: 7721-7732, 2008

32. Yang L, Li N, Wang C, Yu Y, Yuan L, Zhang M and Cao X: Cyclin L2, a novel RNA polymerase II-associated cyclin, is involved in pre-mRNA splicing and induces apoptosis of human hepatocellular carcinoma cells. J Biol Chem 279: 11639-11648, 2004.

33. Araque A, Castillo PE, Manzoni OJ and Tonini R: Synaptic functions of endocannabinoid signaling in health and disease. Neuropharmacology 124: 13-24, 2017.

34. Ohno-Shosaku T and Kano M: Endocannabinoid-mediated retrograde modulation of synaptic transmission. Curr Opin Neurobiol 29: 1-8, 2014

35. Smith-Dijak AI, Sepers MD and Raymond LA: Alterations in synaptic function and plasticity in Huntington disease. J Neurochem 150: 346-365, 2019.

36. Sampson HW, Chaput CD, Brannen J, Probe RA, Guleria RS, Pan J, Baker KM and VanBuren V: Alcohol induced epigenetic perturbations during the inflammatory stage of fracture healing. Exp Biol Med (Maywood) 236: 1389-1401, 2011.

37. Selvamurugan N, He Z, Rifkin D, Dabovic B and Partridge NC: Pulsed electromagnetic field regulates microRNA 21 expression to activate TGF- $\beta$ signaling in human bone marrow stromal cells to enhance osteoblast differentiation. Stem Cells Int 2017: 2450327, 2017.

38. Yoshizuka M, Nakasa T, Kawanishi Y, Hachisuka S, Furuta T, Miyaki S, Adachi N and Ochi M: Inhibition of microRNA-222 expression accelerates bone healing with enhancement of osteogenesis, chondrogenesis, and angiogenesis in a rat refractory fracture model. J Orthop Sci 21: 852-858, 2016.

39. Takahara S, Lee SY, Iwakura T, Oe K, Fukui T, Okumachi E, Waki T, Arakura M, Sakai Y, Nishida K, et al: Altered expression of microRNA during fracture healing in diabetic rats. Bone Joint Res 7: 139-147, 2018

40. Sun P, Zhang D, Huang H, Yu Y, Yang Z, Niu Y and Liu J: MicroRNA-1225-5p acts as a tumor-suppressor in laryngeal cancer via targeting CDC14B. Biol Chem 400: 237-246, 2019.

41. Sun Y, Mei H, Xu C, Tang $H$ and Wei W: Circulating microRNA-339-5p and -21 in plasma as an early detection predictors of lung adenocarcinoma. Pathol Res Pract 214: 119-125, 2018.

42. Li P, Liu H, Li Y, Wang Y, Zhao L and Wang H: MiR-339-5p inhibits lung adenocarcinoma invasion and migration by directly targeting BCL6. Oncol Lett 16: 5785-5790, 2018.

43. Zhang L, Lu XQ, Zhou XQ, Liu QB, Chen L and Cai F: NEAT1 induces osteosarcoma development by modulating the miR-339-5p/TGF- $\beta 1$ pathway. J Cell Physiol 234: 5097-5105, 2019.

44. Pu Y, Zhao F, Cai W, Meng X, Li Y and Cai S: MiR-193a-3p and miR-193a-5p suppress the metastasis of human osteosarcoma cells by down-regulating Rab27B and SRR, respectively. Clin Exp Metastasis 33: 359-372, 2016.

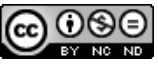

This work is licensed under a Creative Common Attribution-NonCommercial-NoDerivatives 4.0 International (CC BY-NC-ND 4.0) License. 